\title{
Numerical and Experimental Analysis of Gas Flow in a Coaxial Nozzle Applied to Directed Energy Deposition (DED)
}

\author{
J. Grass N., D. Rojas P., G. Barragan D. R. and R. T. Coelho
}

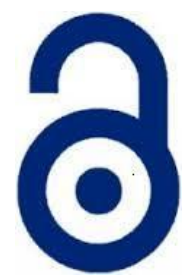

Received: 02 March 2021

Accepted: 30 April 2021

Published: 15 July 2021

Publisher: Deer Hill Publications

(C) 2021 The Author(s)

Creative Commons: CC BY 4.0

\begin{abstract}
Directed Energy Deposition (DED) is an additive manufacturing process, which uses a concentrated laser beam to create a melt pool on the substrate. A nozzle is used to carry metal powder within a gas flow until the melt pool, concentrating the flow at the same point. Coaxial nozzles usually have also a shield gas flow to prevent oxidation and an internal flow to protect the optical system. A right flow configuration must be selected to avoid high turbulence at the nozzle exit, leading to an efficient, inexpensive, and high-quality process. Due to the complexity of the process, CFD - Computer Fluid Dynamics are becoming necessary to understand the behaviour of those gas flows in DED processes. CFD can offer results close to reality and may allow an optimization of the whole nozzle designs, besides selecting the best gas flows for each application.

The present work develops a CFD simulation of the gas flow behaviour in a coaxial nozzle with three internal annular channels (internal, carrier and shield). An initial set of gas flow was selected, based on previous experience of the manufacturer, and then improved. It searches for the low gas consumption, to form a focal point coinciding with the laser focus and a low velocity, which favours the deposition quality. To check the accuracy of the proposed CFD model, experimental measurements of gas velocity were performed and compared with simulated results.
\end{abstract}

Keywords: Additive Manufacturing, DED, CFD

\section{INTRODUCTION}

Additive manufacturing is a process by which 3D object can be produced, starting directly from their 3D CAD. This process is carried out by depositing layer by layer to form the desired geometry, without the need of various manufacturing process and a number of tooling and jigs (1). Amongst existing variety of AM process, Directed Energy Deposition (DED) is one of the best for metal deposition. That is defined as a process in which focused thermal energy source, such as a laser beam, an electron beam or plasms arcs are used to melt metals, which are deposited (2). DED has been widely acclaimed for its ability to create components with complex geometries, customized parts without design restrictions, repairs worn components and modify components already manufactured (3). DED is also considered a disruptive manufacturing method, since it allows the use of different materials in the same process and compared to the traditional manufacturing process, is considered a more profitable process due to the short time it demands and its flexibility (4). In DED process, two forms of raw material can be used; powder and wire. Powder metal is the most used raw material due to its versatility and the fact that most materials can be transformed into powder.

In processes using metal powder, a nozzle is responsible to deliver the powder into the melt pool, using inter gas flows. There are, basically, two types: lateral and coaxial nozzles. Due to its easy fabrication, versatility for working with metal powders and its geometry efficiency, coaxial nozzles have been the most used, lately. The coaxial nozzle can be configured with two or three gas flows. Two flows, use carrier and shield flow. The most recent models contain coaxial annular channels. Designs with three flows use nozzle, carrier and shield channels. The inner channel comes out around the laser beam and it is used to protect the optical system. The carrier gas flows through a middle channel and carries the powder flow to the melt pool. The shield flow comes an outer channel and is used to protect the melt pool against oxidation. (Some author refers to the nozzle gas as axial gas and the shield gas as shape gas) The combination of those three coaxial gas flows is strictly related with the DED process efficiency. They all must

J. Grass N'.凶, D. Rojas P.1, R. T. Coelho' and Barragan G. ${ }^{2}$

'Sao Carlos School Engineering- University of Sao Paulo (EESC-USP)

R. da Reitoria, 374 - Cidade Universitária, Butantã, São Paulo - SP, 05508-220

${ }^{2}$ Grupo de investigación en Ingeniería Aeroespacial, Universidad Pontificia Bolivariana

E-mail: jsgrassn@usp.br 
create a focal point, to deliver the right powder volume to the melt pool, created at the surface in which deposition will occur. Additionally, the powder speed at the melt pool, the combination between feed speed and laser power has to be enough to melt the whole volume of delivered powder. Therefore, to improve the AM process, it is important to develop models to understand the gas flows and demonstrate how the efficiency of the process is affected by them (5).

\section{LITERATURE REVIEW}

Lin (6) performed a numerical analysis, using two types of geometries nozzles. The first with an inward configuration in the inner channel (Fig 1A) and the other with an outward configuration in the same channel (Fig 1B) using powder size between 45 and $100 \mu \mathrm{m}$. It was concluded that the convergence point of powder-gas flow is affected by the coaxial geometry. Using the outward configuration, a widely dispersed stream powder was founded and a convergence point at a distance of $5 \mathrm{~mm}$, which was not very uniform. Using the inward configuration, the focal point could not be visualized, due to the interaction between flows before the coaxial nozzle outlet. In both nozzles it was observed that the powder concentration increased when the gas flow decreased.

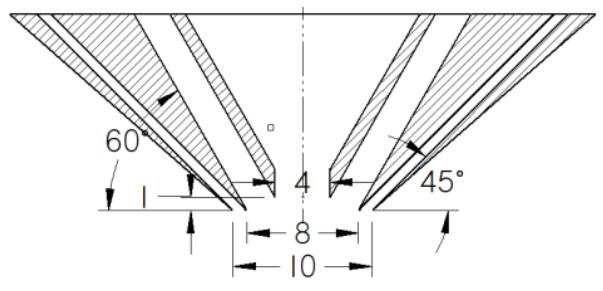

Figure 1: A. inward configuration

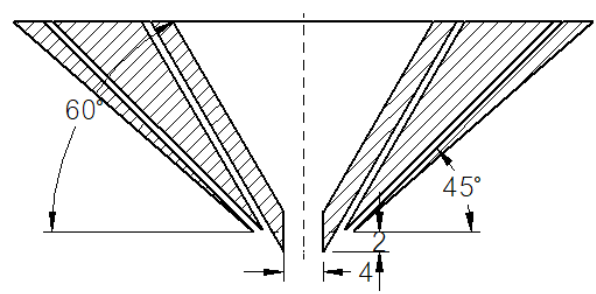

B. outward configuration

In (7) authors carried out a theorical-experimental analysis, measuring the outlet powder velocity in a coaxial nozzle, finding that the highest speed was exactly twice the lowest speed, getting an average of $9.3 \mathrm{~m} / \mathrm{s}$. It was also concluded that due to different gas flows, the particles can have a higher drag after the nozzle outlet.

In (8) authors used the FLUENT ${ }^{\mathrm{TM}}$ software. A numerical analysis was carried out in order to analyse the behaviour of metal powder at different deposition heights, using nitrogen gas and 316L stainless steel particles of $45 \mu \mathrm{m}$, in average. The first simulation was done at free jet, them locating the substrate at a distance of 5,10 and $20 \mathrm{~mm}$ respectively. It was found that as the deposition distance increased, the powder concentration decreasing on the substrate. Authors did recommend the use of depositions distances higher than $10 \mathrm{~mm}$ under those parameters tested.

In (9) authors used a coaxial nozzle with two annular channels. Was setup a standoff $11 \mathrm{~mm}$, gas flow of $3 \mathrm{l} / \mathrm{min}$ in the carrier and $2 \mathrm{l} / \mathrm{min}$ in the shield. A CFD analysis was performed using $\mathrm{k}$ - $\epsilon$ turbulence model to simulate the gas flow. It was found that the convergence point was formed at a distance of $13 \mathrm{~mm}$. Experimentally, it was measured the cross section at the depositions in relation with the powder supply. It was evidenced that the geometry of the coaxial nozzle and the gas flow, offered a process with a 53\% efficiency. Based on these results, the initial coaxial nozzle was redesigned and validate numerical and experimentally, using the same gas flows. Its convergence point was formed at $8 \mathrm{~mm}$ from the nozzle outlet, but this was formed out the laser beam. Carrier gas flow greater than $3 \mathrm{l} / \mathrm{min}$ can create sputter on the substrate and could cause problems to the nozzle. Experimentally it was found that this coaxial nozzle redesigned offered a higher concentration of metal powder with the same gas flows and increase the efficiency of the process to $74.8 \%$.

Koruba, Wall and Reiner in (10) carried out a numerical and experimental analysis of the inert gas on DED process. using ANSYS software to simulate a coaxial nozzle with two annular channels; carrier and shield. Simulating different gas flows, it was founded that as the shield gas flow increases, the focal point is disturbed up to a point where its formation is not able. Using the same gas flows in carrier and shield, only the carrier outlet velocity increases, so the particles will strongly collide with the substrate. Experimentally found that a high flow in carrier result in deposition of higher dilution, deeper HAZ and lower hardness. A high flow in the shield gas, increases the rate of cooling but there was risk of irregularities occurring in the deposition.

In (11) authors used three nozzles with different geometries and configurations. Nozzle A and B with three annular channels: axial, shaping and carrier flows. Nozzle $C$ with two channels: axial, carrier and shielding flows. A numerical and experimental analysis was carried out. Using a pitot tube measurement of the outlet gas velocity in the three nozzles were carried out. In nozzle $C$, it was possible to observe the variation of velocities up to the distance of 11 $\mathrm{mm}$. At a distance of $1 \mathrm{~mm}$, the gas flow was an annular structure, increasing the convergence until forming its convergence point at a distance of $9 \mathrm{~mm}$ and a velocity of $1.85 \mathrm{~m} / \mathrm{s}$ at this point. In nozzle B, it was founded that the outlet velocity was affected by the carrier gas flow, a uniform velocity field was between 11 and $15 \mathrm{~mm}$ and its maximum value was $6.11 \mathrm{~m} / \mathrm{s}$. In nozzle A, the outlet velocity was measured between 1 and $5 \mathrm{~mm}$ and the gas showed a uniform annular structure, reaching a velocity of $20.5 \mathrm{~m} / \mathrm{s}$ and showing that the axial gas flow affect the outlet velocity of the carrier and shield gas. Numerically and using $\mathrm{COMSOL}^{\mathrm{TM}}$ software, it was found that the highest 
velocities were $4.49 \mathrm{~m} / \mathrm{s}, 8.86 \mathrm{~m} / \mathrm{s}$ and $58.9 \mathrm{~m} / \mathrm{s}$ for A, B and C respectively. Estimating measurement errors between $30 \%$ and $50 \%$, which may be associated with atmospheric conditions at the outlet of the nozzle, or errors in the calibration of the pitot tube.

Computed Fluid Dynamics (CFD) is considered the art of replacing the differential equations of the physical aspects of fluids flows with numbers and advancing these numbers in space/time to obtain a final numerical description of the flow field of interest (12). The mesh in CFD simulation is a discrete representation of a geometric domain, which typically includes content and environment form the real world. The creation of the mesh, and the appropriate computational domain has been a challenge, since the complexity of the geometries, the type of flow and the accuracy offered by the different types of meshes must be taken into account. The reliability of the resulted values depends on this. (13)

Bearing that in mind, the present study aims at a numerical analysis of the behaviour of gas flows in a coaxial nozzle applied to DED process. Using a CFD simulation, gas flows were observed on the three channels and different sets of parameters were tested. Selecting one of the flow-sets experimental velocity measurement were performed and compared with simulated results.

\section{CAD AND NUMERICAL MODELLING}

Using a Siemens NX 3D CAD software, a nozzle model was obtained together with a volume below to study the free-flow of gas at outlet (Fig 2).

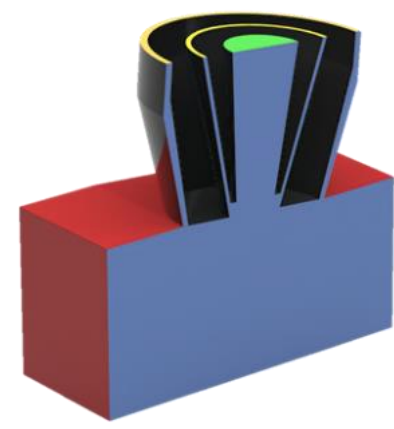

Figure 2: Coaxial Nozzle CAD (Nozzle, Carrier and Shield) with volume at exit.

The volume outside the nozzle has lower limit at $10 \mathrm{~mm}$ below the nozzle, which is the laser focal point and where the substrate has to be positioned. Due to the annular geometry of the nozzle, just half of it was simulated, and was setup a symmetry plane in the computational domain, improving computational efficiency. A refined mesh was used to speed up the simulation and create a better gas flows visualization. In order to no disturb the gas flow, no deposition surface was set below nozzle outlet (fig.2).

The numerical analysis was focused on the dynamics of the gas flow in a DED process. The initial assumption is the following:

- $\quad$ The gas is treated as steady-state turbulent flow with constant velocity distribution in the inlet boundaries

- Boundaries inlet flow have the same gas source

- Constant gas density

- No-slip condition.

Due to the complexity of the geometry for the computational domain, a polyhedral mesh was chosen, which offers a balanced solution to generating complex meshes. This mesh is relatively easy and efficient to construct, as it does not require more surface preparation than the equivalent tetrahedral mesh. Besides, they contain approximately five times fewer cells than a tetrahedral mesh for a given initial surface area.

Simulations were performed using a polyhedral mesh of 2,433,130 elements, being refined in the gas inlets, walls, symmetry plane, and the bottom outlet (Figure 3). Different flow values were simulated in free jet at exit, to understand and observe the gas behaviour after the nozzle outlet and to know the convergence points under different flow conditions. 

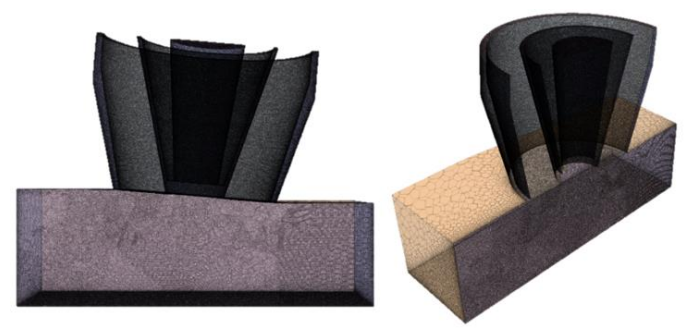

Figure 3: Details of Polyhedral mesh refined used for the computational domain.

Solving gas flows in space-time usually requires very high computational times and for this reasons, different turbulence model are available in the software used most of them based on the Reynolds-Average Navier Stokes (RANS) models (14)

In RANS approximations all fluid instability is considered part of the turbulence and by averaging the non-linearity of the Navier-Stokes equations it results in terms that must be modelled. The complexity of turbulence makes it unlikely that a single RANS model can represent all turbulent flows and for that reason several turbulence models based on RANS were developed. All these considered as engineering approximations and not as physical laws (15) $\mathrm{k}-\epsilon$ is a two-equation turbulence model, which results in the transport equation for the turbulent kinetic energy $\mathrm{K}$ and the turbulent dissipation rate $\epsilon$. In order to determine the turbulent viscosity of the eddy formed by a fluid that is being analysed, it can be defined by the equations below. The turbulent eddy viscosity $\mu t$ is calculated as:

$\mu t=\rho C \mu f \mu k T \quad$ (eq. 1)

Where $\rho$ is the density, $C \mu$ is a model coefficient, $f \mu$ is a damping function and T is the turbulence time scale. The transport equations for the kinetic energy $\mathrm{K}$ and the turbulent dissipation rate $\epsilon$ are:

$$
\begin{aligned}
& \frac{\partial}{\partial t}(\rho k)+\nabla \cdot(\rho k \underline{v})=\nabla \cdot\left[\left(\mu+\frac{\mu_{t}}{\sigma_{k}}\right) \nabla k\right]+P_{k}-\rho\left(\varepsilon-\varepsilon_{0}\right)+S_{k} \\
& \frac{\partial}{\partial t}(\rho \varepsilon)+\nabla \cdot(\rho \varepsilon \underline{v})=\nabla \cdot\left[\left(\mu+\frac{\mu_{t}}{\sigma_{\varepsilon}}\right) \nabla \varepsilon\right]+\frac{1}{T_{e}} C_{\varepsilon 1} P_{\varepsilon}-C_{\varepsilon 2} f_{2} \rho\left(\frac{\varepsilon}{T_{e}}-\frac{\varepsilon_{0}}{T_{0}}\right)+S_{\varepsilon} \text { (eq. 3) }
\end{aligned}
$$

Where $\underline{v}$ is the mean velocity, $\mu$ is the dynamic viscosity. $\sigma_{k}, \sigma_{\varepsilon}, C_{\varepsilon 1}$, and $C_{\varepsilon 2}$ are model coefficients. $P_{k}$ and $\rho \varepsilon$ are production terms. $f_{2}$ is a damping function. $S_{k}$ and $S_{\varepsilon}$ are the user-specified source terms.

Variable $\varepsilon_{0}$ is the ambient turbulence in the source terms that counteracts turbulence delay. The possibility to impose an ambient source term also leads to the definition of a specific time-scale $T_{0}$, that is defined as:

$T_{0}=\max \left(\frac{k_{0}}{\varepsilon_{0}}, C_{t} \sqrt{\frac{v}{\varepsilon_{0}}}\right)$ (eq. 4)

where $C_{t}$ is a model coefficient.

The turbulent production is given by:

$G_{k}=\mu_{t} S^{2}-\frac{2}{3} \rho k \nabla \cdot \underline{v}-\frac{2}{3} \mu_{t}(\nabla \cdot \underline{v})^{2}$

The buoyancy production is given by:

$G_{b}=\beta \frac{\mu_{t}}{P r_{t}}(\nabla \underline{T} \cdot g)$

$\beta$ is the coefficient of the thermal expansion. For the constant density flows using the boussinesq approximation, $\beta$ is user specified, $P r_{t}$ is the turbulent Prandtl number, $T$ is the gravitational vector [19].

The numerical solution was carried out using a Siemens STAR-CCM+ ${ }^{\mathrm{TM}}$. It was considered an inert gas atmosphere in the computational domain, which neglects the air-gas mixture. Inert gas was set with the argon industrial properties $\rho=1.69 \frac{\mathrm{kg}}{\mathrm{m}^{3}}$. 
Initially, Condition A was simulated as that recommended by the manufacturer of the deposition head. Then, different sets were simulated always towards minimizing gas consumption and improving deposition quality. Table 1 shows the whole set of combinations simulated.

Condition B used the minimum value for the nozzle, tested to save some argon gas and to observe its effect on the overall flow configuration. By analysing the results up to that point, intermediate low values for nozzle and shield gas were tested together with a slightly higher value for carrier, making condition C. Following conditions D, E and $\mathrm{F}$, were selected in attempts to achieve two simultaneous conditions: stablish a convergence point for the carrier flow and minimizing the velocity at these points.

Table 1: Conditions to numerical analysis

\begin{tabular}{|c|c|c|c|}
\hline Conditions & \multicolumn{1}{c}{$\begin{array}{c}\text { Nozzle (lt/ } \\
\text { min) }\end{array}$} & $\begin{array}{c}\text { Carrier } \\
(\mathrm{lt} / \mathrm{min})\end{array}$ & $\begin{array}{c}\text { Shield } \\
(\mathrm{lt} / \mathrm{min})\end{array}$ \\
\hline A & 6 & 4 & 10 \\
\hline B & 2 & 4 & 10 \\
\hline C & 4 & 5 & 8 \\
\hline D & 3 & 6 & 9 \\
\hline E & 5 & 7 & 6 \\
\hline F & 4 & 4 & 8 \\
\hline
\end{tabular}

\section{EXPERIMENTAL SETUPS}

The pitot tube was clamped in a 3 jaw chuck positioned at the origin of the $X, Y$ machine axe. The chuck center was found using the RENISHAW OMP 60 OPTICAL probe supplied with the machining center, ROMI hybrid D800 model.

The pitot tube was positioned directly into the fluid flow and flow velocity was measured. Using Bernoulli principle pressure was measured using a micromanometer TSI 8705 and related with speed using equation (7):

$v=\sqrt{\frac{2\left(P_{\text {stag }}-P\right)}{\rho}}($ eq.7)

The pitot tube used has a single internal diameter of $1 \mathrm{~mm}$ and was connected with micromanometer by a plastic tube. (Fig.4)

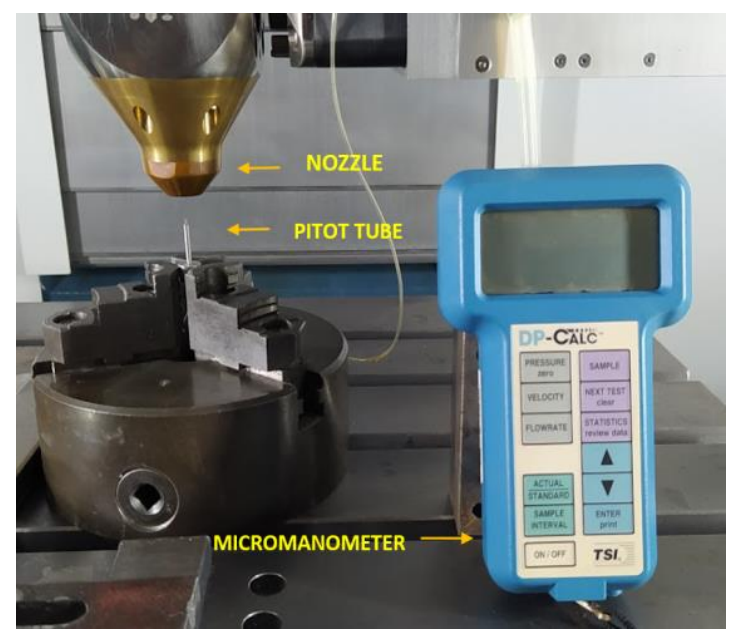

Figure 4: Setup experimental test

Flow velocities were measured in $1 \mathrm{~mm}$ intervals over the $X$ and $Y$ axe. an $X-Y$ on the $Z$ direction, the intervals were at every $2 \mathrm{~mm}$, from 0 to $10 \mathrm{~mm}$ below the nozzle. At each measuring point, a time of 20 seconds was given for the argon flow and the micro manometer measurement to stabilize. Figure 5 shows the set up for measurements.

The experimental data were further processed using an interpolation function in MATLAB ${ }^{\mathrm{TM}}$ and later plotted in $3 D$ graphs for better examinations. 


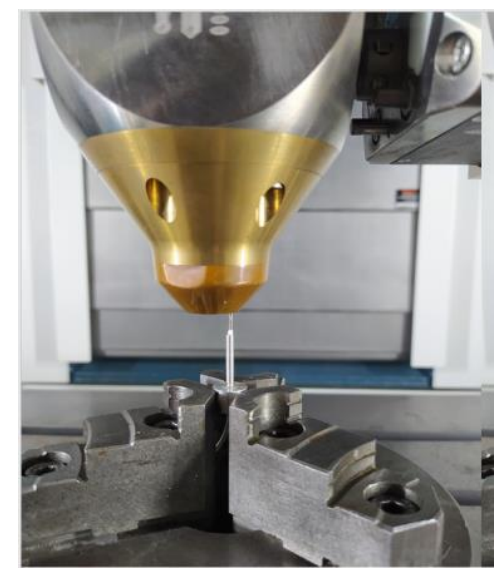

Figure 5: Starting position of pitot tube in $\mathrm{Y}$ axes.

\section{RESULTS AND DISCUSSION}

\subsection{Numerical Results}

Figure 6 shows the gas flow simulation for condition A. The gas streams do not form a clear convergence point at 10 $\mathrm{mm}$ where laser focal point and the substrate is supposed to be located. It can be noticed that upon reaching the substrate, the gas flow velocity is approx. $7 \mathrm{~m} / \mathrm{s}$, which can give an idea of what the velocity of the powder would be upon reaching the melt pool at the substrate. Although the velocity is not very high and there is no convergence point at $10 \mathrm{~mm}$ below the nozzle, deposition is possible with that set of flows.

To reduce gas consumption and reduce outlet velocity, condition B was configured using the nozzle minimum flow rate (Figure 7). This configuration shows a convergence point at approx. $5.41 \mathrm{~mm}$ from the outlet nozzle and a flow velocity at the substrate of approx. $3.14 \mathrm{~m} / \mathrm{s}$. This would be a better set of flows because it achieves a convergence point and with lower velocity at the substrate. However, the nozzle flow at $2 \mathrm{l} / \mathrm{min}$ could risk the optical system for long deposition times and such condition should never be used in real tests.

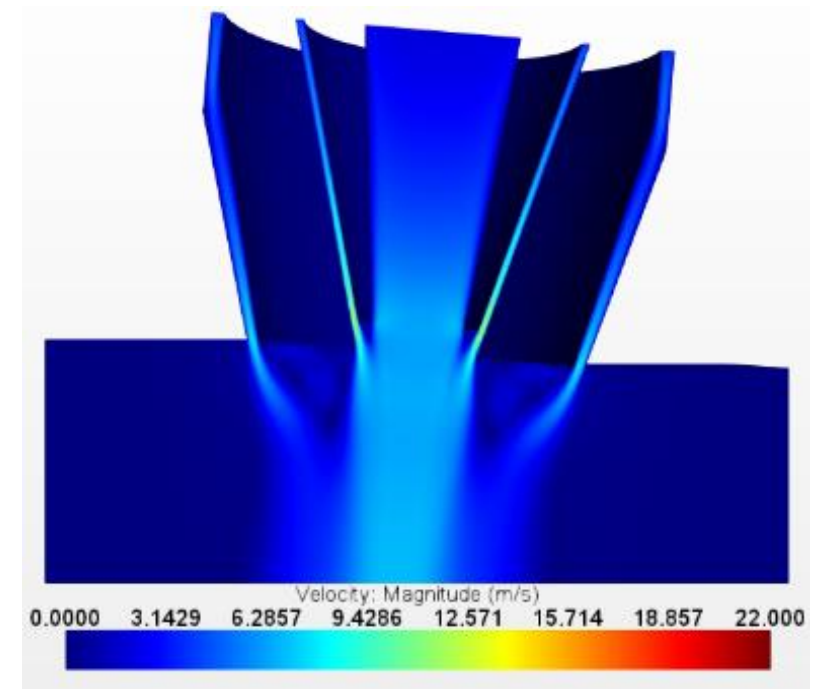

Figure 6: Simulation using condition A

Continuing with reducing the gas flow but avoiding the risk for the optical system, an increase in the gas nozzle and carrier was performed, which was compensated with a reduction in the shielding gas flow, this being condition $\mathrm{C}$, shown in Fig 8. The gas velocity of the carrier gas is considerably increased, which gives a coaxial shape to the gas flow and prevents the formation of the convergence point at $10 \mathrm{~mm}$. It was also observed that the velocity of the gas at the substrate is around $7.5 \mathrm{~m} / \mathrm{s}$. This is a comparable to Condition A.

Condition D is shown in Fig 9 in which one can see a better convergence point at $7.36 \mathrm{~mm}$ with velocity of approx. $8.5 \mathrm{~m} / \mathrm{s}$ at this point and maintained until reaching the substrate. Velocity at the substrate is higher than Condition $\mathrm{B}$, but still comparable with the others before. 


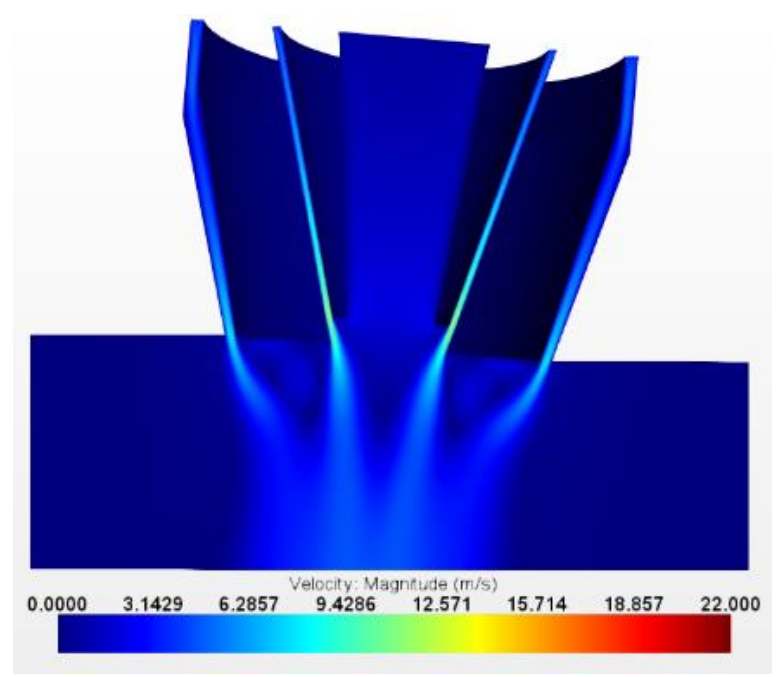

Figure 7: Simulation using condition B

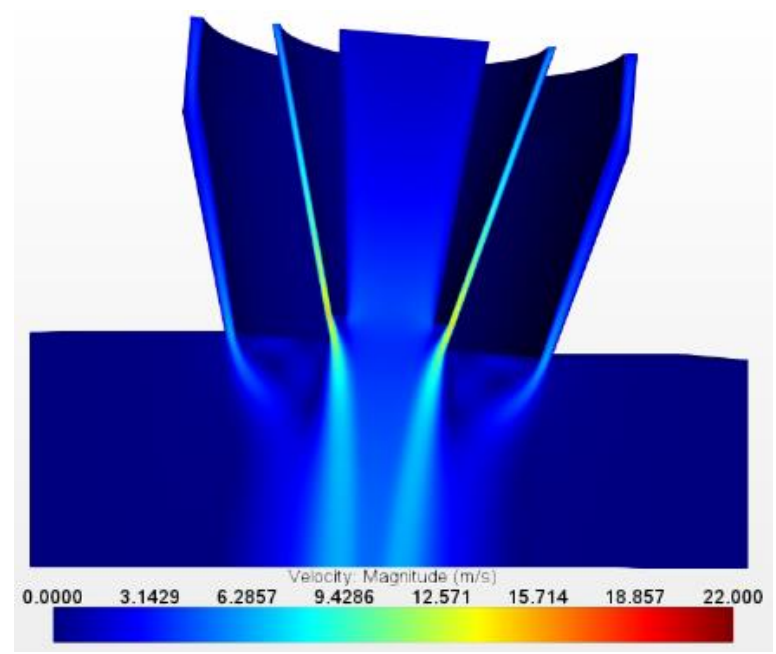

Figure 8: Simulation using condition $C$

In an attempt to form a clear focal point, an increase in all flows was made. To compensate, a drastic reduction in the gas shield was made to evaluate its behaviour under minimum flow. Such simulation is shown in Fig 10. In this configuration, high exit velocities were obtained in the carrier, preventing the formation of the convergence point before $10 \mathrm{~mm}$. Condition $\mathrm{E}$ may cause some sputter in case of deposition. It was also evidenced that when the gas flow shielding is very low, it may lead to oxidation preventing the use for practical depositions.

Condition F, shown in Fig. 11 was intended to reduce the carrier velocity, test the same amount of gas flow in nozzle, and a slight increase the shielding flow. The formation of a focal point below $10 \mathrm{~mm}$ was not achieved, but the gas consumption was significantly reduced. The velocity when reaches the substrate is between 2.7 and $4.12 \mathrm{~m} / \mathrm{s}$, which can be considered a very low velocity and could offer smooth depositions and improve the process efficiency. However, experimental confirmation will have to be done. 


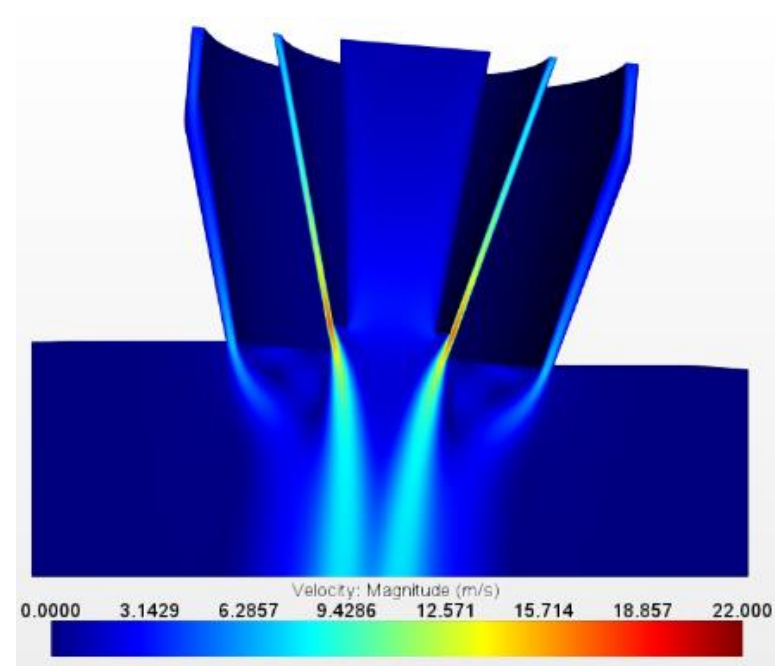

Figure 9: Simulation using condition D

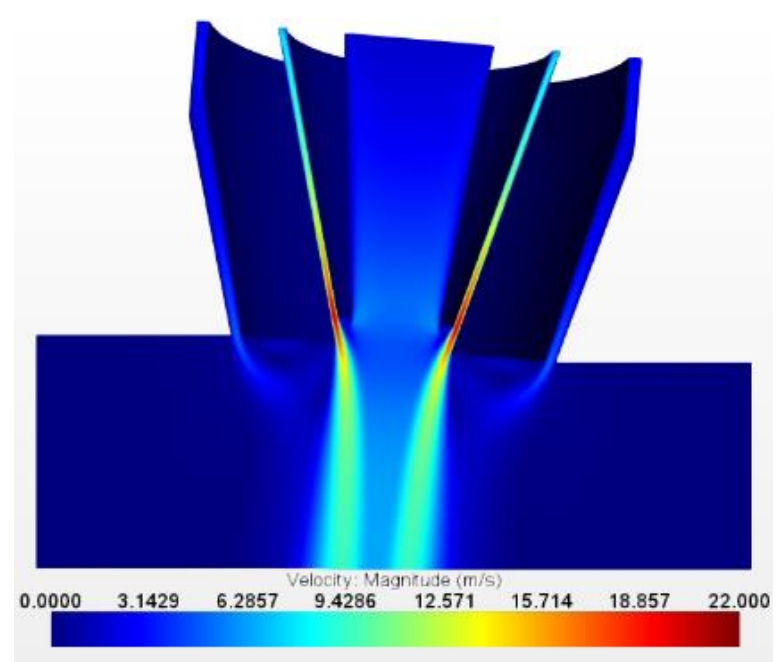

Figure 10: Simulation using condition E

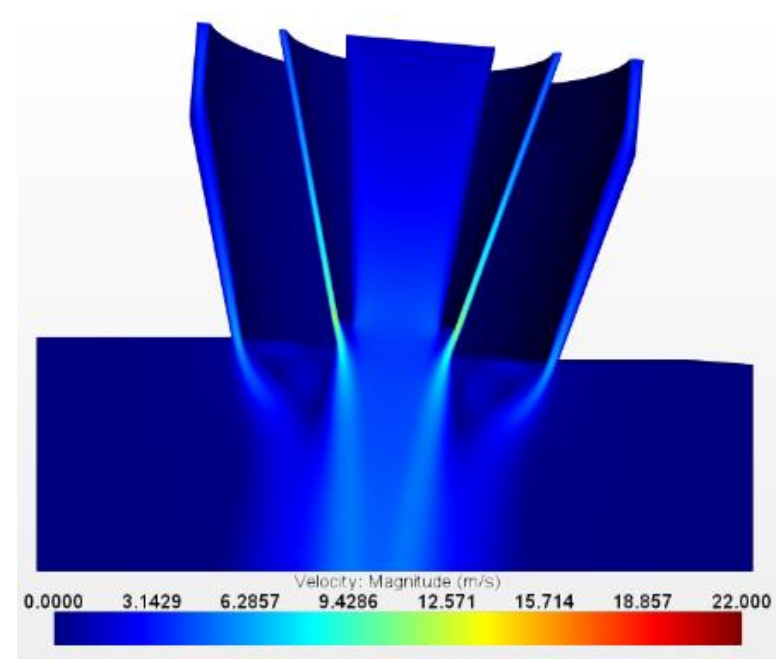

Figure 11: Simulation using condition F 


\subsection{Experimental Results}

Figures 12 to 17 shows gas flow measurements in a 3D shape at $0,2,4,6,8$ and $10 \mathrm{~mm}$ form the nozzle, performed for Condition E. It can be observed that velocity is not uniform around the nozzle. In addition, maximum velocity values are different at extremes. The same pattern was found with increasing distance from the nozzle. Besides that, the actual concentration of the gas flow tends to be off centre, at $10 \mathrm{~mm}$ by about $1 \mathrm{~mm}$ in each axis.
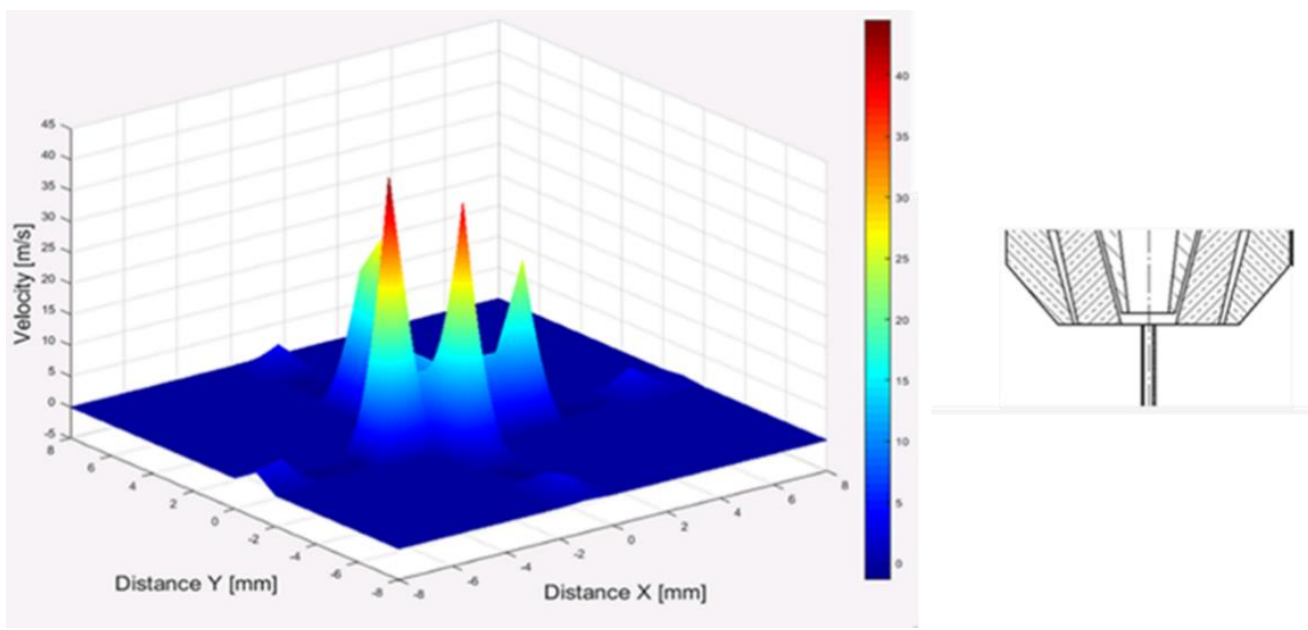

Figure 12: gas velocity measured at $0 \mathrm{~mm}$ from the nozzle
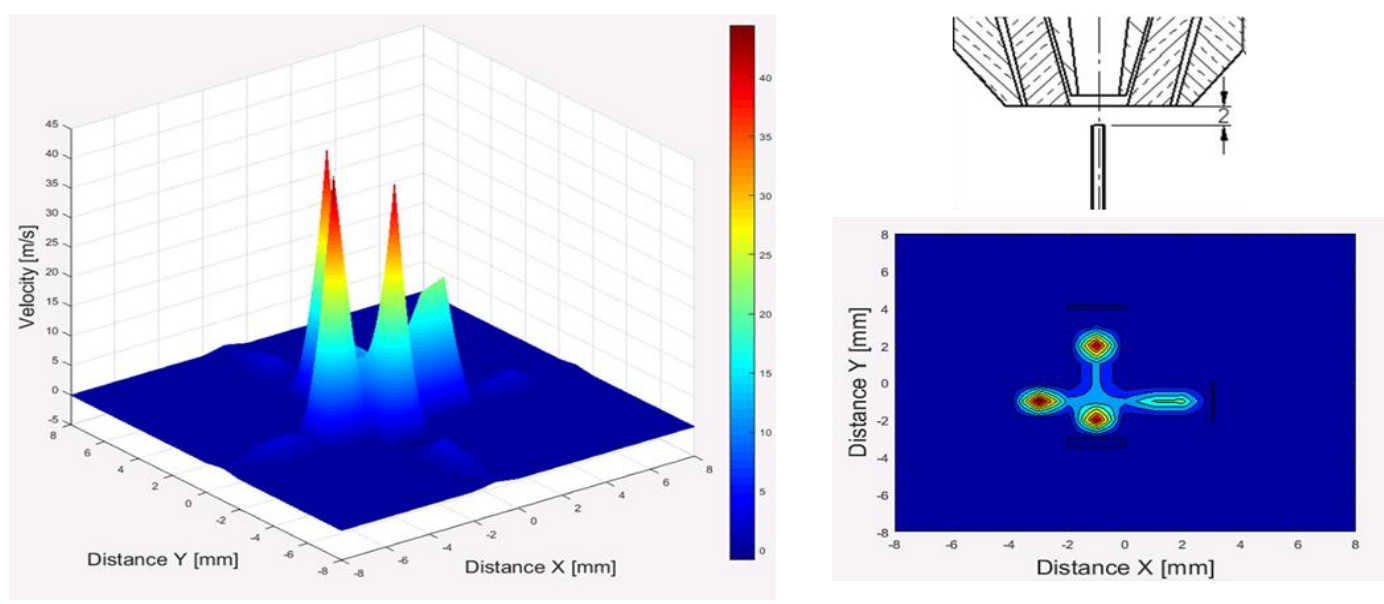

Figure 13: gas velocity measured at $2 \mathrm{~mm}$ from the nozzle 


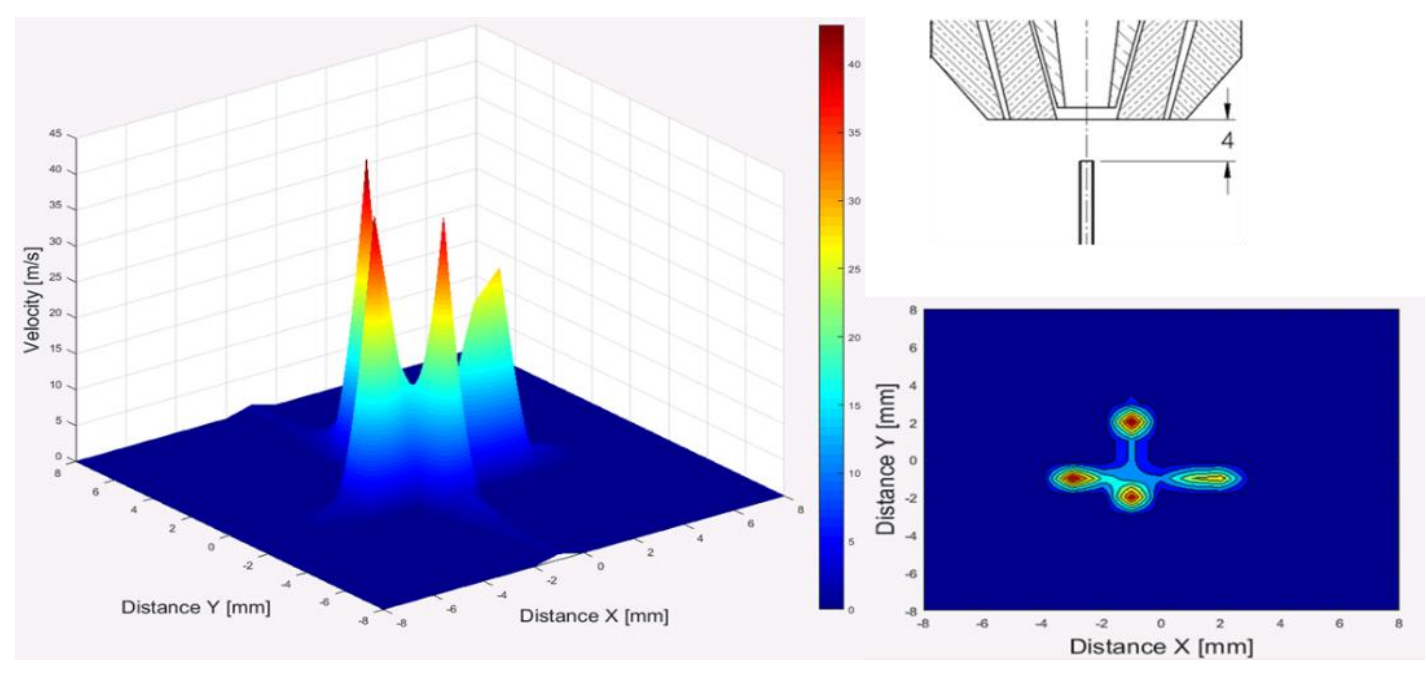

Figure 14: gas velocity measured at $4 \mathrm{~mm}$ from the nozzle
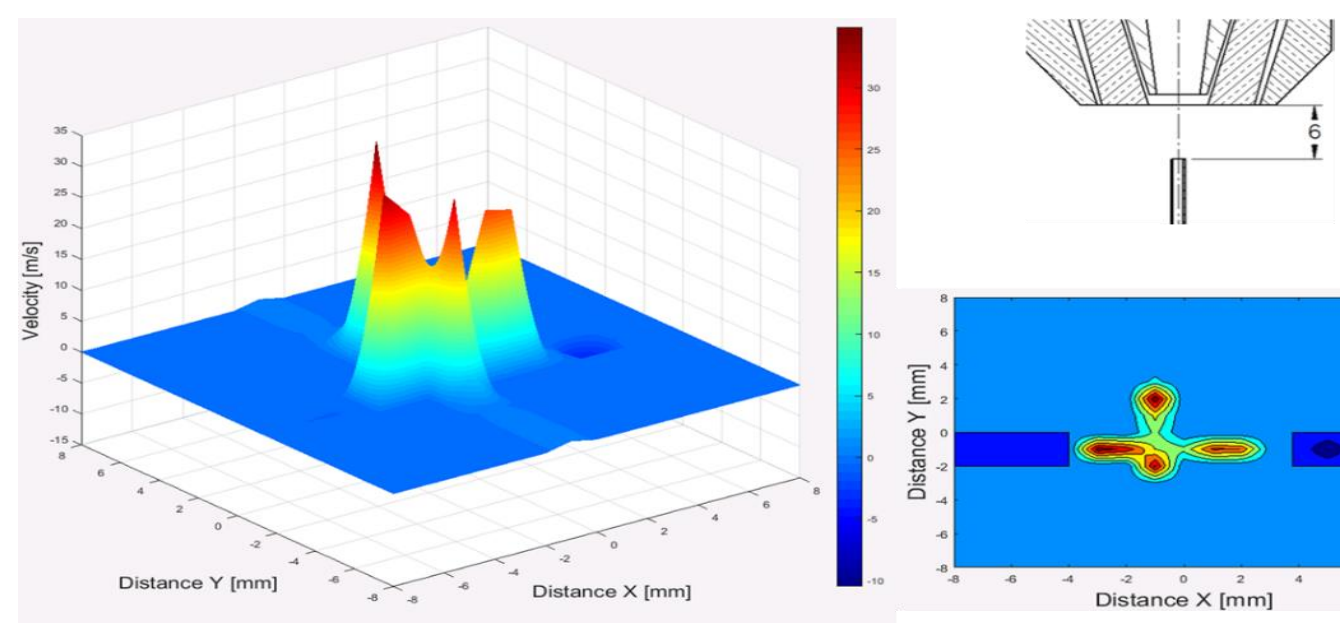

Figure 15: gas velocity measured at $6 \mathrm{~mm}$ from the nozzle

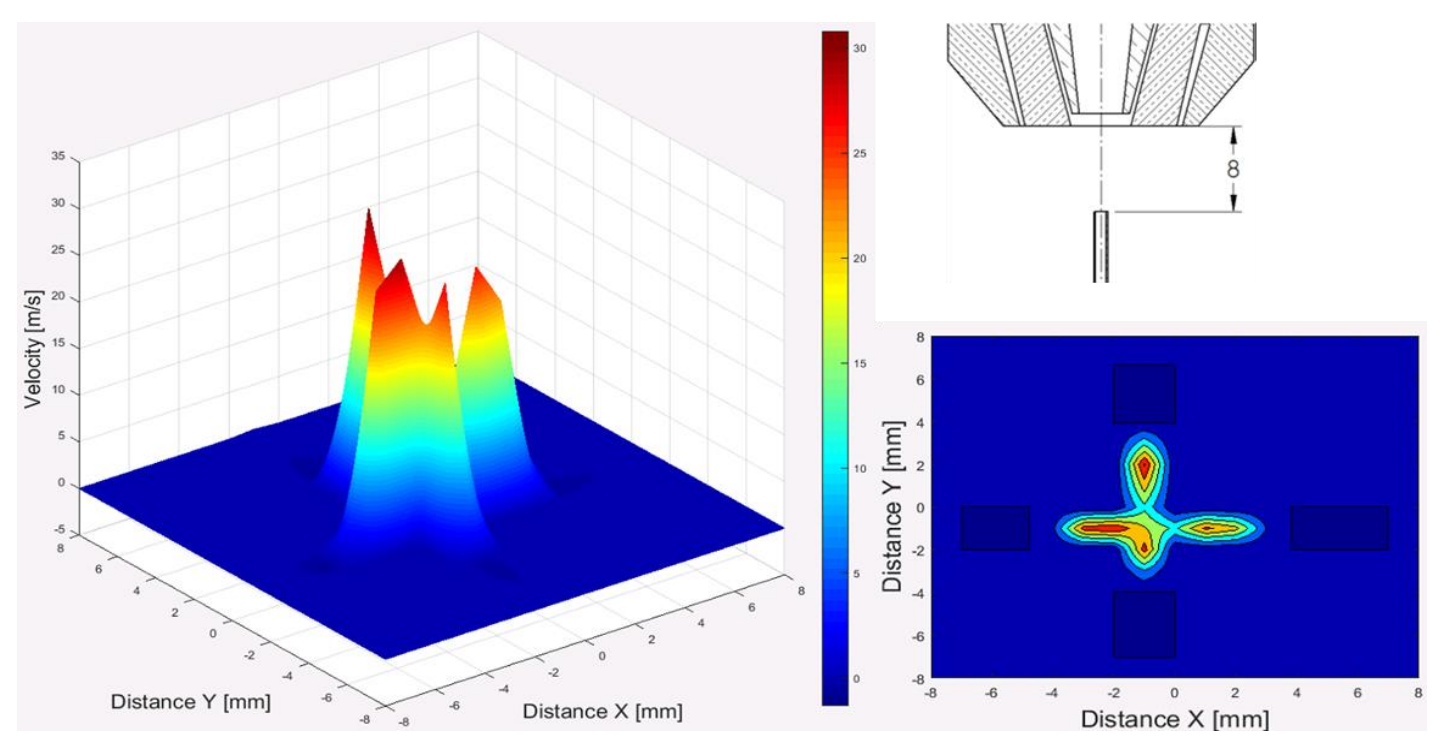

Figure 16: gas velocity measured at $8 \mathrm{~mm}$ from the nozzle 


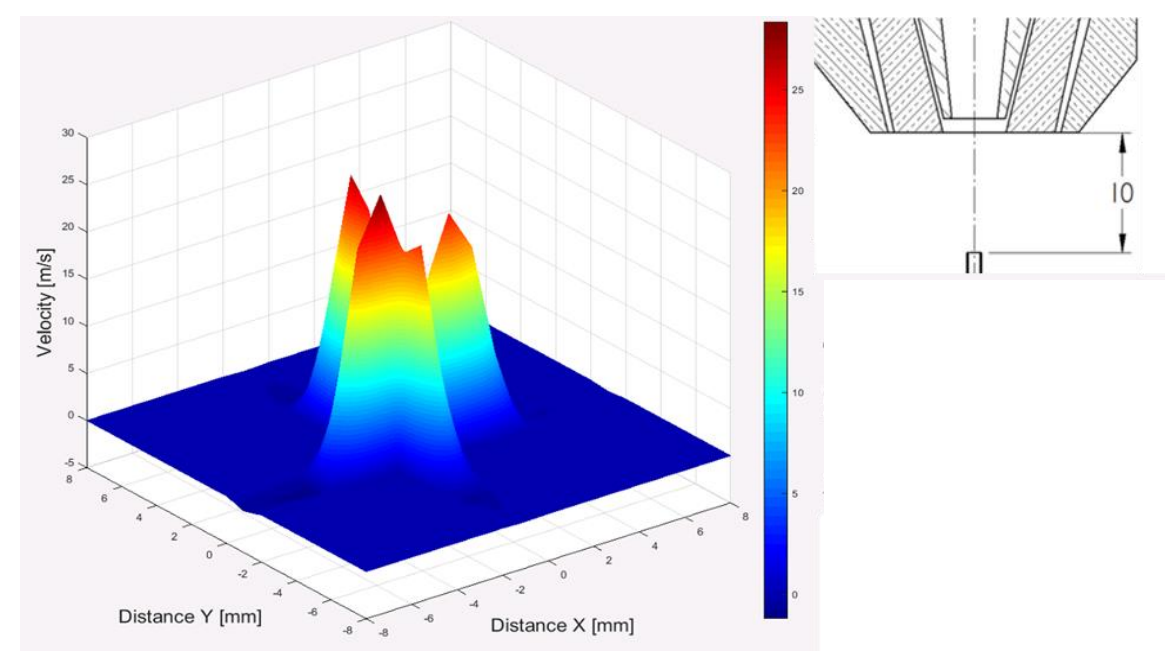

Figure 17: gas velocity measured at $10 \mathrm{~mm}$ from the nozzle

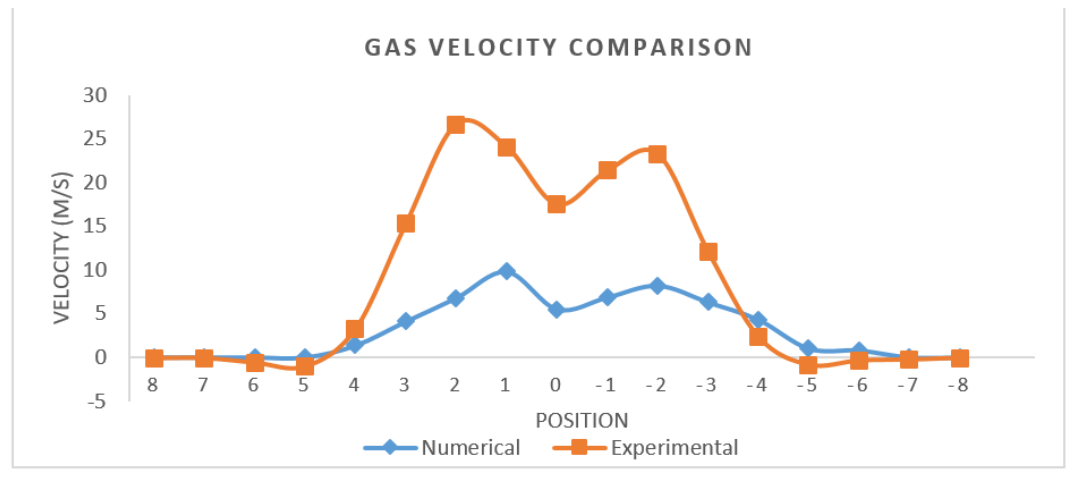

Figure 18: A comparison of numerical and experimental gas velocity values at $10 \mathrm{~mm}$ distance from the nozzle.

Some clear differences of velocities comparing numerical analysis (Fig. 10) and experimental measurement (Fig 17) can be observed. However, both results describe a velocity field with the same shape, i.e., describing a hat top function. Flow velocity measurements using a Pitot tube, based on the Bernoulli equation is more appropriate for flows with higher Reynolds number (16). In low Reynolds number, the viscosity has a strong effect on the flow pattern, therefore limiting the use of the Bernoulli equation. However, this equation is based on an invaluable model, which makes these measurements sufficient for the description of velocity fields (17).

The major differences between CFD and experimental results, can also be due to calibration errors, taking into account that it had not internal aspect ratio diameters, a possible bad location of the static pressure intake and the difference in the densities air-argon presents in outlet coaxial nozzle, could cause errors in the measurements.

Such result indicates that CFD computational models can be used for describing gas flows behaviour at the exit of coaxial nozzles and, therefore, can be a valuable tool for improving nozzle efficiency in DED processes.

\section{CONLSUSIONS}

A CFD model was developed and used for analysing gas flows in a 3-channel coaxial nozzle applied to DED process. Different gas flow sets were simulated. An experimental work was also carried out to compare simulated and experimental flow velocities. From all those studies the following conclusions were possible:

- CFD analysis indicated that gas flows are sensitive parameters and they may affect the deposition quality and process efficiency. Small variations in the inlet gas parameter, significantly affect outlet velocities and can drastically gas flow field, particularly focal point formation and carrier speed at the substrate.

- It was found that Condition A (Fig. 6) offers an excellent set of gas flow with a focal point and relatively low velocity at the substrate. Gas consumption is slightly high and could be lowered.

- Condition B (Fig 7) showed some advantages in terms of gas consumption and focal point formation, but it is not recommended because optical system could suffer in long time depositions. 
- Regarding all the other conditions tested, it can be stated that it is not recommending using a shield gas lower than $8 \mathrm{l} / \mathrm{min}$ for a height deposition of $10 \mathrm{~mm}$, or higher. Shield outlet velocity is easily affected by the carrier gas flow creating turbulence and it may not reach the substrate with sufficient speed to produce the expected protective atmosphere.

- Although experimental velocity values were higher than those obtained from CFD simulation, the speed fields were similar indicating the same tendency. Bearing that in mind, CDF can be a valuable tool for studying and improving nozzle designs and the DED process as a whole.

\section{ACKNOWLEDGEMENT}

This study was financed in part by the Coordenação de Aperfeiçoamento Pessoal de Nível Superior - Brasil (CAPES) - Finance Code 001; São Paulo Research foundation (FAPESP); Laboratory for Advanced Processes and Sustainability (LAPRAS).

\section{REFERENCES}

1. Gebhardt A, Kessler J, Thurn L. Basics of 3D Printing Technology. In: 3D Printing [Internet]. München: Carl Hanser Verlag $\mathrm{GmbH} \&$ Co. KG; 2018. p. 1-32. Available from: https://www.hanserelibrary.com/doi/10.3139/9781569907030.001

2. ASTM. Standard Terminology for Additve Manufacturing Technologies. ASTM Int. 2013 Sep 9;

3. Kamal M, Rizza $G$. Design for metal additive manufacturing for aerospace applications. In: Additive Manufacturing for the Aerospace Industry [Internet]. Elsevier; 2019. p. 67-86. Available from: http://dx.doi.org/10.1016/B978-0-12-814062-8.00005-4

4. Saboori A, Aversa A, Marchese G, Biamino S, Lombardi M, Fino P. Application of directed energy depositionbased additive manufacturing in repair. Appl Sci. 2019;9(16).

5. Vilar R. Laser cladding. J Laser Appl [Internet]. 1st ed. 1999 Apr;11(2):64-79. Available from: http://lia.scitation.org/doi/10.2351/1.521888

6. Lin J. Numerical simulation of the focused powder streams in coaxial laser cladding. J Mater Process Technol [Internet]. $2000 \quad$ Sep;105(1-2):17-23. Available from: https://linkinghub.elsevier.com/retrieve/pii/S0924013600005847

7. Kovalev OB, Zaitsev A V., Novichenko D, Smurov I. Theoretical and Experimental Investigation of Gas Flows, Powder Transport and Heating in Coaxial Laser Direct Metal Deposition (DMD) Process. J Therm Spray Technol [Internet]. 2011 Mar 21;20(3):465-78. Available from: http://link.springer.com/10.1007/s11666-010-9539-3

8. Zhu G, Li D, Zhang A, Tang Y. Numerical simulation of metallic powder flow in a coaxial nozzle in laser direct metal deposition. Opt Laser Technol [Internet]. 2011 Feb;43(1):106-13. Available from: http://dx.doi.org/10.1016/j.optlastec.2010.05.012

9. Koike R, Takemura S, Kakinuma Y, Kondo M. Enhancement of powder supply efficiency in directed energy deposition based on gas-solid multiphase-flow simulation. Procedia CIRP [Internet]. 2018;78:133-7. Available from: https://doi.org/10.1016/j.procir.2018.09.061

10. Koruba P, Wall K, Reiner J. Influence of processing gases in laser cladding based on simulation analysis and experimental tests. Procedia CIRP [Internet]. 2018;74:719-23. Available from: https://doi.org/10.1016/j.procir.2018.08.025

11. Ferreira E, Dal M, Colin C, Marion G, Gorny C, Courapied D, et al. Experimental and numerical analysis of gas/powder flow for different LMD nozzles. Metals (Basel). 2020;10(5):1-20.

12. Wendt JF, Anderson JD, Degroote J, Degrez G, Dick E, Grundmann R, et al. Computational Fluid Dynamics [Internet]. Wendt JF, editor. Computational Fluid Dynamics. Berlin, Heidelberg: Springer Berlin Heidelberg; 2009. 1-332 p. Available from: http://link.springer.com/10.1007/978-3-540-85056-4

13. plm.automation.siemens. Meshing [Internet]. 2020. [cited 2020 Jul 25]. Available from: https://documentation.thesteveportal.plm.automation.siemens.com/starccmplus_latest_en/index.html?param $=$ hw9F1\&authLoc $=$ https://thesteveportal.plm.automation.siemens.com/AuthoriseRedirect\#page/STARCCMP \%2FGUID-112029FC-12E0-426F-85FC-4198DE68A414.html\%23

14. plm.automation.siemens. Trimmed Mesher [Internet]. 2020 [cited 2020 Jul 25]. Available from: https://documentation.thesteveportal.plm.automation.siemens.com/starccmplus_latest_en/index.html\#page/S TARCCMP\%2FGUID-DB165A77-56AD-4854-A970-C84BF90B68AD.html\%23

15. Ferziger JH, M.peric. Computational Methods For Fluid Dynamics. third, rev. Berlin; Heidelberg; New York; Barcelona; Hong Kong; London; Milan; Paris; Tokyo: Springer Berlin Heidelberg; 2002.

16. Spelay RB, Adane KF, Sanders RS, Sumner RJ, Gillies RG. The effect of low Reynolds number flows on pitot tube measurements. Flow Meas Instrum [Internet]. 2015;45:247-54. Available from: http://www.sciencedirect.com/science/article/pii/S095559861500062X

17. Boetcher SKS, Sparrow EM. Limitations of the standard Bernoulli equation method for evaluating Pitot/impact tube data. Int J Heat Mass Transf [Internet]. 2007;50(3):782-8. Available from: http://www.sciencedirect.com/science/article/pii/S0017931006001396 